\title{
Amplitude modulated Bloch oscillations of photon probability distribution in cavity-atom system
}

\author{
G Zhang ${ }^{1}, \mathbf{W} \mathbf{H} \mathbf{H u}^{1,2}, \mathrm{Z}_{\text {Song }}{ }^{1}$ \\ ${ }^{1}$ School of Physics, Nankai University, Tianjin 300071, China \\ ${ }^{2}$ Beijing Computational Science Research Center, Beijing 100084, China \\ E-mail: songtc@nankai.edu.cn
}

\begin{abstract}
We study the dynamics of the Rabi Hamiltonian in the medium coupling regime with $|g / \omega| \sim 0.07$, where $g$ is atom-field coupling constant, $\omega$ is the field frequency, for the quantum state with average photon number $\bar{n} \sim 10^{4}$. We map the original Hamiltonian to an effective one, which describes a tight-binding chain subjected to a staggered linear potential. It is shown that the photon probability distribution of a Gaussian-type state exhibits the amplitude modulated Bloch oscillations (BOs), which is a superposition of two conventional BOs with a half-BO-period delay between them and is essentially another type of Bloch-Zener oscillation. The probability transition between the two BOs can be controlled and suppressed by the ratio $g \sqrt{\bar{n}} / \omega$, as well as in-phase resonant oscillating atomic frequency $\Omega(t)$, leading to multiple zero-transition points.
\end{abstract}

PACS numbers: 42.50.Pq, 03.65.Xp, 42.50.-p, 


\section{Introduction}

The interaction of matter and light is one of the fundamental processes occurring in nature. Its elemental constituent is the coupling between single atoms and photons. The quantum Rabi model considers a two-level atom coupled to a quantized field, describing the simplest interaction between quantum light and matter. It was used to describe the interaction between a rapidly varying, weak magnetic field and nuclear spin [1, 2]. Now it applies to a variety of physical systems with the two-level atom, being trapped ions, quantum dots, and superconducting qubits. Such systems can be exploited as a building block for quantum information processing and other potential applications to future quantum technologies [3].

Although the quantum Rabi model seems relatively simple and was declared to be solved recently [4, 5], the dynamics are actually quite complicated, depending on the system parameters and the initial state [6]. The rotating wave approximation (RWA) is justified in the strong coupling regime (but the average photon number can not be too large) and results in the Jaynes-Cummings (JC) model [7], which predicts nonclassical phenomena, such as revivals of the initial excited state of the atom [8, 9, 10]. It is well known that an atom-cavity system undergoes the Rabi oscillations. In the case of the single cavity mode being initially prepared in a coherent state with large average photon number, although the oscillations experience the collapse-and-revival, the photon probability distribution remains unchanged in the framework of the JC model. The question is whether it is true if the original Rabi model is considered, or what will be observed in experiment. With strong couplings in the solid-state-cavityQED [11], the failure of RWA has relighted the interest on the quantum Rabi model [4, 6, 12, 13, 14, 15, 16, 17, 18, 19].

In this paper, we study the dynamics of the Rabi Hamiltonian in the medium coupling regime with $|g / \omega| \sim 0.07$, where $g$ is atom-field coupling constant, $\omega$ is the field frequency, for the quantum state with average photon number $\bar{n} \sim 10^{4}$. The effective coupling constant becomes $|g \sqrt{\bar{n}}| \sim 7 \omega$, which goes beyond the RWA. Within this parameter regime, the original Hamiltonian is mapped to an effective one, which describes a uniform tight-binding chain subjected to a staggered linear potential. An approximate solution suggests that the photon number distribution of a Gaussiantype state exhibits the amplitude modulated Bloch oscillations (BOs), which is a superposition of two conventional BOs with a half-BO-period delay between them. This phenomenon essentially belongs to Bloch-Zener oscillation in the strong tunneling limit $[20,21,22,23,24,25]$. We find that the probability transition between the two BOs can be controlled and suppressed by the ratio $g \sqrt{\bar{n}} / \omega$, as well as resonant oscillating atomic frequency $\Omega(t)$, leading to multiple zero-transition points. A numerical simulation of dynamics in the Rabi model confirms this prediction.

This paper is organized as follows. In Section 2, we propose the effective Hamiltonian in the concerned parameter regime. In Section 3, we present the approximate solution of the effective Hamiltonian, based on which the dynamics of 
a local state is investigated. Section 4 is dedicated to a numerical simulation in the original Rabi model, focusing the control of the probability transition between two BOs by the atomic transition frequency. Finally, we give a summary and discussion in Section 5.

\section{Model and equivalent Hamiltonian}

We start by considering the single-mode atom-cavity model whose Hamiltonian can be written as

$$
H=\omega \hat{a}^{\dagger} \hat{a}+\frac{\Omega}{2} \hat{\sigma}_{x}+g \hat{\sigma}_{z}\left(\hat{a}^{\dagger}+\hat{a}\right),
$$

where $\omega$ and $\Omega$ are the field and atomic transition frequencies, respectively, and $g$ is the coupling constant. $\hat{a}^{\dagger}(\hat{a})$ is the creation (annihilation) operator of the light field, while $\hat{\sigma}_{x}=|e\rangle\langle e|-| g\rangle\left\langle g\left|, \hat{\sigma}_{z}=\right| e\right\rangle\langle g|+| g\rangle\langle e|$ are atomic operators, where $|g\rangle$ and $|e\rangle$ denote the ground and excited atomic states, respectively. There is an approximation which has been developed, the rotating-wave approximation (RWA) [26, 27] under the condition $|g \sqrt{\bar{n}}| \ll \omega$.

In this paper, we consider the case with the medium coupling regime with $|g / \omega| \sim$ 0.07 and the average photon number $\bar{n} \sim 10^{4}$. Such a parameter regime is accessible in experiments [28, 29, 30]. The aim of this paper is to investigate the dynamics of a particular initial state with a Gaussian-type photon number distribution, which allows us to obtain the approximate analytical result from the Hamiltonian (1).

Since Hamiltionian (11) is parity-conserving for the excitation number

$$
\mathcal{N}=a^{\dagger} a+\frac{1}{2}\left(\hat{\sigma}_{x}+1\right)
$$

it can be written in two independent equivalent Hamiltonian $H_{\mathrm{eq}}^{\mathrm{e}}\left(H_{\mathrm{eq}}^{\mathrm{o}}\right)$ in the bases with even (odd) excitation number:

$$
\begin{aligned}
H_{\mathrm{eq}}^{\lambda}= & g \sum_{i=0}^{\infty} \sqrt{i+1}\left(|i\rangle_{\lambda}\langle i+1|+\text { H.c. }\right) \\
& +\sum_{i=0}^{\infty}\left[\frac{(-1)^{\gamma_{\lambda}+i}}{2} \Omega+i \omega\right]|i\rangle_{\lambda}\langle i|,
\end{aligned}
$$

where $\lambda=\mathrm{e}, \mathrm{o}$ and $\gamma_{\mathrm{e}}=1, \gamma_{\mathrm{o}}=0$. This is schematically illustrated in Fig. 1. The equivalence between $H$ and $H_{\text {eq }}^{\lambda}$ is based on the mapping of the corresponding basis: for even invariant subspace we have $\{|\mathrm{g}, 0\rangle,|\mathrm{e}, 1\rangle,|\mathrm{g}, 2\rangle,|\mathrm{e}, 3\rangle,|\mathrm{g}, 4\rangle, \cdots\}$ $\longrightarrow\left\{|i\rangle_{\mathrm{e}}\right\}$, where $i=0,1,2, \cdots$, while for odd invariant subspace we have $\{|\mathrm{e}, 0\rangle,|\mathrm{g}, 1\rangle,|\mathrm{e}, 2\rangle,|\mathrm{g}, 3\rangle,|\mathrm{e}, 4\rangle, \cdots\} \longrightarrow\left\{|i\rangle_{\mathrm{o}}\right\}[6]$. Note that $H_{\mathrm{eq}}^{\lambda}$ is a standard tightbinding chain with coordinate-dependent nearest neighbor (NN) hopping strength and on-site potential. It is easy to find out that, within the region of large excitationnumber limit $i \gg 1$, when the initial state is local near $\bar{n} \sim 10^{4}$, we can take truncated approximation to $H_{\mathrm{eq}}^{\lambda}$. In this case, the expansion of the coupling strength in equation (3) is $\sqrt{i}=\sqrt{\bar{n}}+\frac{i-\bar{n}}{2 \sqrt{\bar{n}}}-\frac{(i-\bar{n})^{2}}{8 \bar{n}^{3 / 2}}+\ldots$. We take the first term only, then $\sqrt{i} \approx \sqrt{\bar{n}}$; therefore, 


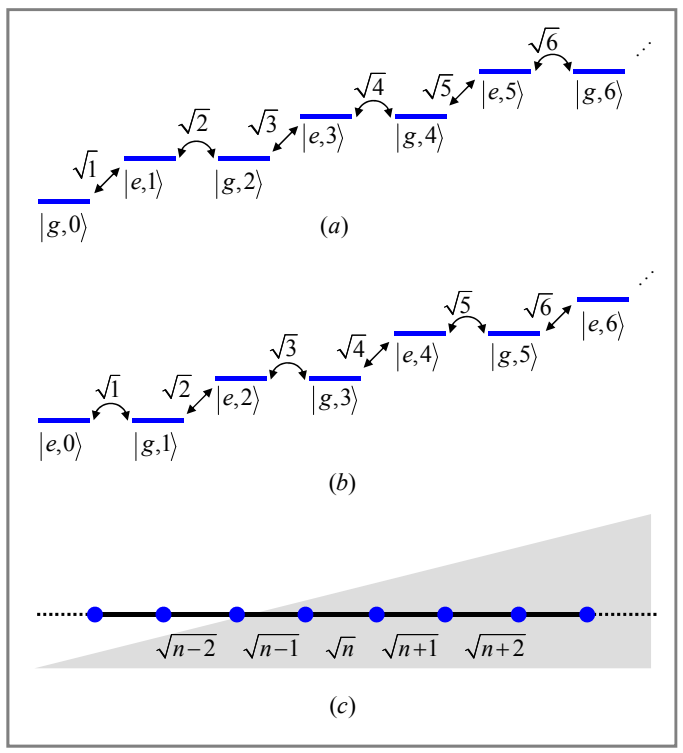

Figure 1. (Color online) The atom-cavity level structure used for the mapping to a tight-binding chain, and the implementation of Bloch oscillations in the photon-number space. Atom-cavity level diagram showing the lower-energy states for a two-level atom of transition frequency $\Omega$ coupled (with single-photon Rabi frequency $g$ ) to a single mode cavity field of frequency $\omega$, with $\Omega=\omega$. (a) and (b) correspond to even- and odd-parity cases, respectively. The transition strengths are in units of $g$. (c) For large photon region $n \gg 1$, both even- and odd-parity cases can be mapped to a tightbinding chain with uniform NN hopping strength $(\sqrt{n+1} \simeq \sqrt{n})$ and linear potential (as represented by gray shadow) approximately, which allows the occurrence of Bloch oscillations.

$H_{\text {eq }}^{\lambda}$ can be approximately equivalent to a tight-binding chain with uniform NN hopping strength and staggered linear potential. In the following, we neglect the label $\lambda$ in the effective Hamiltonian for simplicity and demonstrate that such a discrete system admits the existence of a robust BO [31, 32, 33].

\section{Approximate formalism}

In this section, we will introduce an approximate formalism for the approximate solution of the effective Hamiltonian and investigate the dynamical behavior as an application.

\subsection{Effective Hamiltonian}

We rewrite the effective Hamiltonian in the form

$$
\begin{aligned}
& H_{\mathrm{eff}}=H_{0}+H_{1} \\
& H_{0}=g \sqrt{\bar{n}} \sum_{i=0}^{N}(|i\rangle\langle i+1|+\text { H.c. })+\omega \sum_{i=0}^{N}\left(i-\frac{N}{2}\right)|i\rangle\langle i|,
\end{aligned}
$$


Amplitude modulated Bloch oscillations of photon probability distribution in cavity-atom system5

$$
H_{1}=\frac{\Omega}{2} \sum_{i=0}^{N}(-1)^{i}|i\rangle\langle i|,
$$

where $\bar{n}$ is the average number of photon and can be regarded as a constant in the context of the problem we concern in this paper. This Hamiltonian is equivalent to (3) for the dynamics of a local state within the region $i \gg 1$. Notice that the hamiltonian $H_{\text {eff }}$ is nothing but the tight-binding Hamiltonian to describe a single particle subjected to a staggered linear potential, which has been well studied in previous literatures [20]. It is demonstrated that the Bloch-Zener oscillations with a single period occurs in the parameter regime $|g / \omega| \sim 0.2$ and $\Omega=6.734 \omega$. In this paper, we revisit the same model within the parameter regime $|g / \omega| \sim 0.07$ and $\Omega=\omega$, which is accessible in experiment [28, 29, 30]. We will show that the dynamics undergoes Bloch-Zener oscillations characterized by two periods.

In the absence of term $H_{1}$, it is a standard model which admits the existence of Wannier-Stark localization and Bloch oscillations, and has been studied extensively [34, 35, 36, 37. Now the question is: what happens to the dynamics of the model in Eq. (4). The basic idea is to consider the term $H_{1}$ as a perturbation. According to the theory of Wannier-Stark localization [31, 32], the solution of $H_{0}$ has the form

$$
H_{0}\left|\psi_{m}\right\rangle=E_{m}^{0}\left|\psi_{m}\right\rangle
$$

with

$$
E_{m}^{0}=\omega m-\frac{N}{2} \omega,
$$

and

$$
\begin{aligned}
& \left|\psi_{m}\right\rangle=\frac{1}{\sqrt{N}} \sum_{k} \exp \left[-i\left(m k+\frac{L}{2} \sin k\right)\right]|k\rangle \\
& |k\rangle=\frac{1}{\sqrt{N}} \sum_{l=1}^{N} e^{i k l}|l\rangle, m=0, \pm 1, \pm 2, \ldots
\end{aligned}
$$

In real space, it reads

$$
\left|\psi_{m}\right\rangle=\sum_{l=1}^{2 N} \mathrm{~J}_{l-m}\left(\frac{L}{2}\right)|l\rangle .
$$

where $L=-4 g \sqrt{\bar{n}} / \omega$, is the spatial extent of a single Bloch wave packet oscillation [32], and $g$ is negative.

In order to examine the effect of $H_{1}$ on the dynamics of the system, it is convenient to work in the interaction picture. The propagator of the whole Hamiltonian $H$ in the interaction picture is obtained by the unitary transformation

$$
H_{\mathrm{I}}(t)=e^{\mathrm{i} H_{0} t} H_{1} e^{-\mathrm{i} H_{0} t} .
$$

The propagator is

$$
U^{I}(t, 0)=\mathcal{T} \exp \left[-\mathrm{i} \int_{0}^{t} H_{I}\left(t^{\prime}\right) d t^{\prime}\right],
$$


where $\mathcal{T}$ is the time order operator. In the basis of states $\left\{\left|\psi_{n}\right\rangle\right\}$, one obtains

$$
\left\langle\psi_{m}\left|H_{\mathrm{I}}(t)\right| \psi_{n}\right\rangle=e^{\mathrm{i}(m-n) \omega t} \frac{\Omega}{2}(-1)^{m} \mathrm{~J}_{n-m}(L) .
$$

We will reduce it by the following two steps. Firstly, we can neglect rapidly-oscillating terms in $e^{\mathrm{i} n \omega t}$ for $n \geq 2$. Secondly, we note that $\left|\mathrm{J}_{1}(x)\right| \ll\left|\mathrm{J}_{0}(x)\right|$ when $x$ is around the zeros of $\left|J_{1}(x)\right|,\left|J_{1}\left(x_{0}\right)\right|=0$. Then when the parameter $L \simeq x_{0}$, with $x_{0}=2.41,5.53$, $\ldots$, one can drop the term of $\left\langle\psi_{m}\left|H_{\mathrm{I}}(t)\right| \psi_{m \pm 1}\right\rangle$. Therefore the approximation and the feature of the Bessel function lead to the following approximate expression

$$
\left\langle\psi_{m}\left|H_{\mathrm{I}}(t)\right| \psi_{n}\right\rangle \approx \frac{\Omega}{2}(-1)^{m} \mathrm{~J}_{0}(L) \delta_{m n},
$$

i.e., state $\left|\psi_{n}\right\rangle$ is the simultaneous eigenstate of time-dependent Hamiltonian $H_{\mathrm{I}}(t)$. Then in the basis of states $\left\{\left|\psi_{n}\right\rangle\right\}$, the propagator in Eq. (13) is reduced to

$$
U_{m n}^{I}(t, 0) \approx \delta_{m n} \exp \left[-\mathrm{i} \int_{0}^{t}\left\langle\psi_{m}\left|H_{\mathrm{I}}\left(t^{\prime}\right)\right| \psi_{n}\right\rangle d t^{\prime}\right] .
$$

The corresponding propagator $U(t, 0)$ in Schrödinger picture is in the form

$$
U_{m n}(t, 0) \approx \delta_{m n} \exp \left\{-\mathrm{i}\left[m \omega+(-1)^{m} \gamma\right] t\right\},
$$

where

$$
\gamma=\frac{\Omega}{2} \mathrm{~J}_{0}(L) .
$$

\subsection{Dynamics}

To clarify the feature of the dynamics, we consider the time evolution of an arbitrary state. We note that any state can be decomposed into even and odd-parity portions, which are spanned by the state $\left|\psi_{n}\right\rangle$ with even or odd $n$, respectively. It shows that the parity is conserved during the time evolution under an arbitrary (either odd or even) $H$ within the approximate framework. We see that the even and odd-parity parts of quantum state evolve independently. Furthermore, the appearance of the term $H$ only contributes an overall phase on each parts. It is presumable that the profile of the evolved state is determined by the factor $\gamma$ in a simply manner. It plays a central role in the emerging interference phenomena of two subwaves with different parity.

Actually, for a given initial state

$$
|\psi(0)\rangle=\sum_{n} f_{n}\left|\psi_{n}\right\rangle
$$

we have

$$
\begin{aligned}
|\psi(t)\rangle & =U(t, 0)|\psi(0)\rangle \\
& =e^{-\mathrm{i} \gamma t} \sum_{n} e^{-\mathrm{i} 2 n \omega t} f_{2 n}\left|\psi_{2 n}\right\rangle+e^{\mathrm{i} \gamma t} \sum_{n} e^{-\mathrm{i}(2 n+1) \omega t} f_{2 n+1}\left|\psi_{2 n+1}\right\rangle .
\end{aligned}
$$

In order to demonstrate the physical picture of state $|\psi(t)\rangle$, we consider the time evolution of the same initial state under the free Hamiltonian $H_{0}$, which can be written as

$$
\left|\psi_{0}(t)\right\rangle=e^{-\mathrm{i} H_{0} t}|\psi(0)\rangle=\sum_{n} e^{-\mathrm{i} n \omega t} f_{n}\left|\psi_{n}\right\rangle
$$


It is turned out that $\left|\psi_{0}(t)\right\rangle$ exhibits standard Bloch oscillation with period $T_{B}=2 \pi / \omega$ for a local initial state. Furthermore, a straightforward derivation shows that

$$
|\psi(t)\rangle=\cos (\gamma t)\left|\psi_{0}(t)\right\rangle-\mathrm{i} \sin (\gamma t)\left|\psi_{0}\left(t+\frac{T_{B}}{2}\right)\right\rangle \text {. }
$$

It shows that state $|\psi(t)\rangle$ can be regarded as the superposition of two evolved states under the Hamiltonian $H_{0}$. In order to get a physical picture of the phenomenon, we introduce an operator $\hat{B}_{\pi}$ which relates two states $\left|\psi_{0}(t)\right\rangle$ and $\left|\psi_{0}\left(t+\frac{T_{B}}{2}\right)\right\rangle$ in the following way

$$
\hat{B}_{\pi}\left|\psi_{0}(t)\right\rangle=\left|\psi_{0}\left(t+\frac{T_{B}}{2}\right)\right\rangle .
$$

Obviously, $\hat{B}_{\pi}$ can be expressed in term of $H_{0}$ as

$$
\hat{B}_{\pi}=e^{-\mathrm{i} H_{0} T_{B} / 2} .
$$

The physical meaning of operator $\hat{B}_{\pi}$ becomes clear if we apply it on the state $|k\rangle$. A direct derivation shows that

$$
\hat{B}_{\pi}|k\rangle=\exp [\mathrm{i} L \sin k]|k+\pi\rangle .
$$

It indicates that operator $\hat{B}_{\pi}$ is nothing but the $\pi$-boost operator that shifts all momentum states by $\pi$ with an extra phase factor. Then if we consider the initial state as a Gaussian wave packet $\left|\phi\left(n_{0}, k_{0}\right)\right\rangle$ with momentum $k_{0}$ and the center position $n_{0}$, approximately, it evolves as

$$
\begin{aligned}
|\psi(t)\rangle= & \cos (\gamma t) e^{-\mathrm{i} H_{0} t}\left|\phi\left(n_{0}, k_{0}\right)\right\rangle \\
& -\mathrm{i} \sin (\gamma t) e^{-\mathrm{i} H_{0} t} \exp \left(\mathrm{i} L \sin k_{0}\right)\left|\phi\left(n_{0}+n_{\pi}, k_{0}+\pi\right)\right\rangle
\end{aligned}
$$

where $n_{\pi}=-L \cos k_{0}$. Here the Gaussian wave packet has the form

$$
\left|\phi\left(n_{0}, k_{0}\right)\right\rangle=\frac{1}{\sqrt{R}} \sum_{n=0}^{\infty} e^{-\frac{\alpha^{2}}{2}\left(n-n_{0}\right)^{2}} e^{\mathrm{i} k_{0} n}|n\rangle,
$$

where $R$ is the normalization factor and $\alpha$ determines the half width of the wave packet, $\Delta=2 \sqrt{2 \ln 2} / \alpha$ in the case of $\alpha \ll 1$. We can see that the evolved state represents the superposition of Bloch oscillations of two wave packets with a $n_{\pi}$-shifted in position and a $\pi$-shifted in momentum. The amplitude of each oscillation is modulated with sinusoidal time dependence.

Particularly, we see that in the case of the parameters satisfying the equation

$$
\gamma n T_{B}=\frac{\pi}{2},(n \in \mathbb{N})
$$

the wave packet $\left|\phi\left(n_{0}, k_{0}\right)\right\rangle$ is separated as two equal-probability ones, $\left|\phi\left(n_{0}, k_{0}\right)\right\rangle$ and its counterpart $\left|\phi\left(n_{0}+n_{\pi}, k_{0}+\pi\right)\right\rangle$, at instant $t=\frac{n T_{B}}{2}$. On the other hand, we see that $|\psi(t)\rangle$ and $\left|\psi_{0}(t)\right\rangle$ coalesce with each other at instants $t=t_{n}$, where

$$
t_{n}=\frac{n \pi}{\gamma},(n \in \mathbb{N}) .
$$




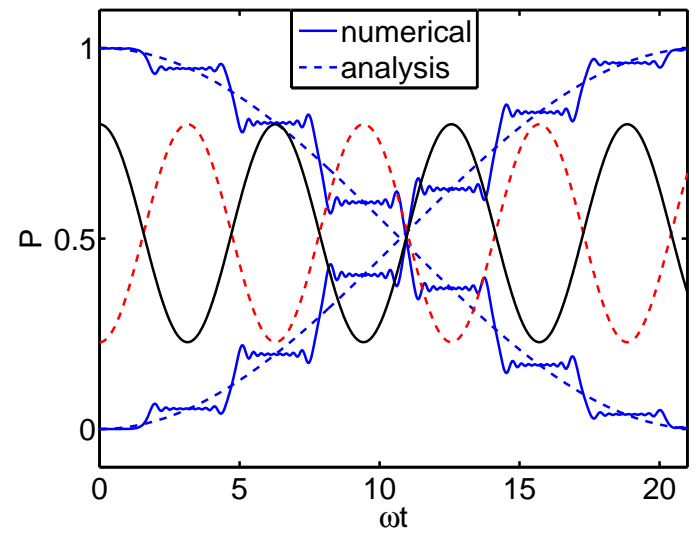

Figure 2. (Color online) Probability transition between two BOs obtained by approximate method presented in this paper and numerical simulation. The initial wavepacket $|\psi(0)\rangle=\left|\phi\left(n_{0}, 0\right)\right\rangle$ with $n_{0}=1.01 \times 10^{4}$ and $\alpha=0.1$ is involved in the Hamiltonian $H_{\text {eff }}$ with $\Omega=\omega$ and $L=28.89$. The black solid line and red dashed line are the shapes of Eq. (30), while blue dashed lines represent the Eq. (31). The solid blue lines indicate the numerical results for $P_{a}(t)$ and $P_{b}(t)$, which is in accord with our prediction that $\Omega$ can lead to the probability transition in the special intervals.

To exemplify these features, we give the center positions $n_{a}(t), n_{b}(t)$ and corresponding probabilities $P_{a}(t), P_{b}(t)$ of two wave packets as function of time for the initial state $|\psi(0)\rangle=\left|\phi\left(n_{0}, 0\right)\right\rangle$ as the form

$$
n_{a}(t)=n_{b}+L \cos (\omega t)=n_{0}-L \sin ^{2}(\omega t / 2)
$$

and

$$
P_{a}(t)=1-P_{b}(t)=\cos ^{2}(\gamma t)
$$

which is evolved in the Hamiltonian $H_{\text {eff }}$ in Eq. (4) with constant $\Omega$.

However, it is worthy to point out that the above conclusions are obtained within the approximate framework, which we will see from the following analysis. One can consider the mechanism of the modulated BOs in an alternative way. For a wave packet $\left|\phi\left(n_{0}, k_{0}\right)\right\rangle$ at a certain location, the action of $H_{0}$ is to drive it to evolve as a Bloch oscillation, while the action of $H_{1}$ is to make a transition from $\left|\phi\left(n_{0}, k_{0}\right)\right\rangle$ to its counterpart, i.e.,

$$
H_{1}\left|\phi\left(n_{0}, k_{0}\right)\right\rangle \rightarrow\left|\phi\left(n_{0}, k_{0}+\pi\right)\right\rangle .
$$

Meanwhile, the energy difference between two wave packets

$$
\begin{aligned}
\Delta E= & \frac{\Omega}{2}\left\langle\phi\left(n_{0}, k_{0}\right)\left|H_{0}\right| \phi\left(n_{0}, k_{0}\right)\right\rangle \\
& -\frac{\Omega}{2}\left\langle\phi\left(n_{0}, k_{0}+\pi\right)\left|H_{0}\right| \phi\left(n_{0}, k_{0}+\pi\right)\right\rangle \\
= & 2 \Omega g \sqrt{\bar{n}} \cos k_{0}
\end{aligned}
$$

suppresses this process. It indicates that only under the condition $k_{0} \approx \pm \pi / 2$, such a transition has a higher probability to occur due to the fact $\Delta E \approx 0$. We note that 
the condition $k_{0} \approx \pm \pi / 2$ is satisfied when $e^{-\mathrm{i} H_{0} t}\left|\phi\left(n_{0}, k_{0}\right)\right\rangle$ and $e^{-\mathrm{i} H_{0} t}\left|\phi\left(n_{0}, k_{0}+\pi\right)\right\rangle$ overlap with each other in real space. The underlying mechanism of this phenomenon is that $H_{1}$ is a local interaction operator. To demonstrate this point, numerical simulation is performed by exact diagonalization for the truncated matrix of the Hamiltonian $H_{\text {eq }}$ rather than $H_{\text {eff }}$. The probabilities $P_{a}(t)$ and $P_{b}(t)$ are computed by $\left|\left\langle\psi(0) e^{\mathrm{i} H_{0} t} e^{-\mathrm{i} H_{\mathrm{eq}} t} \mid \psi(0)\right\rangle\right|^{2}$ and $\left|\left\langle\psi(0) e^{\mathrm{i} H_{0}\left(t+T_{B} / 2\right)} e^{-\mathrm{i} H_{\mathrm{eq}} t} \mid \psi(0)\right\rangle\right|^{2}$ respectively. In Fig. 2 we plot the center positions and the probability distributions of two evolved wave packets $e^{-\mathrm{i} H_{0} t}|\psi(0)\rangle$ and $e^{-\mathrm{i} H_{0}\left(t+T_{B} / 2\right)}|\psi(0)\rangle$, obtained by analytical expression in Eqs. (30) and (31), and the numerical simulation.

It shows that the probability distributions are step-like as functions of time and the probability exchange happens when two wave packets meet together. When they meet each other for the first time, the splitting of a Gaussian wave packet can be observed. It also indicates that our analytical results, Eqs. (30) and (31), is the time-averaged approximation for the probability transition.

A Gaussian wave packet with the center position $\bar{n}$ and the half width $\Delta=$ $2 \sqrt{2 \ln 2} / \alpha=23.5$, considering the evolution which has been discussed above, can be regarded as being local within a spatial extent, $D=L+6 / \alpha$, where $L=-4 g \sqrt{\bar{n}} / \omega$. When $\bar{n} \sim 10^{4}, L \sim 28, D<100$, now we let $D=100$; in this case, if we want to satisfy $|(\sqrt{i}-\sqrt{\bar{n}}) / \sqrt{\bar{n}}|<5 \%$ for $i \in D, \bar{n} \sim 10^{3}$ is mandatory at least.

In the next section, the validity of the prediction will be investigated in the Rabi system by numerical simulation. We will quantitatively evaluate the extent of approximation of the above analysis.

\section{Controllable dynamics of cavity-atom system}

Now we apply the obtained results to a concrete case and then demonstrate the dynamic property of the system. We investigate the time evolution of the wave packet in the single-mode atom-cavity model. As mentioned above, the dynamics of local state in large excitation number region is equivalent to that of the Hamiltonian in Eq. (44). We will show that the modulation of the Bloch oscillations can be controlled by the coupling constant and the energy level of the atom, which can be adjusted via external field.

As an application of the obtained result, let us take a simple case as an example. Without loss of generality, we assume that the initial state is in the form

$$
|\psi(0)\rangle=\frac{|g\rangle+|e\rangle}{\sqrt{2 R}} \sum_{n} e^{-\frac{\alpha^{2}}{2}(n-\bar{n})^{2}}|n\rangle .
$$

Transforming the basis $\{|\mathrm{g}, n\rangle,|\mathrm{e}, n\rangle\}$ to $\left\{|i\rangle_{\mathrm{e}}\right\}$ and $\left\{|i\rangle_{\mathrm{o}}\right\}$, we have

$$
|\psi(0)\rangle=\frac{1}{\sqrt{2 R}} \sum_{\lambda=\mathrm{e}, \mathrm{o}} \sum_{i=0}^{\infty} e^{-\frac{\alpha^{2}}{2}(i-\bar{n})^{2}}|i\rangle_{\lambda},
$$

which corresponds to the superposition of two independent stationary wave packets in two chains $H_{\text {eff }}^{\lambda}$ with $\lambda=\mathrm{e}$, o, respectively. According to the above analysis, this state 
Amplitude modulated Bloch oscillations of photon probability distribution in cavity-atom system 10

evolves to

$$
\begin{aligned}
|\psi(t)\rangle= & \frac{1}{\sqrt{2 R}} \sum_{n}^{\infty} e^{-\mathrm{i} n \omega t}\left\{\left[\vartheta_{t} \cos (\gamma t)+\mathrm{i} \vartheta_{t+T_{B} / 2} \sin (\gamma t)\right]|g, n\rangle\right. \\
& \left.+\left[\vartheta_{t} \cos (\gamma t)-\mathrm{i} \vartheta_{t+T_{B} / 2} \sin (\gamma t)\right]|e, n\rangle\right\},
\end{aligned}
$$

where

$$
\begin{aligned}
& \vartheta_{t}=\exp \left[-\mathrm{i} \mu-\frac{\alpha^{2}}{2}\left(n-n_{t}\right)^{2}\right], \\
& \mu=-\frac{L}{2} \sin (\omega t), \\
& n_{t}=\bar{n}-L \sin ^{2}\left(\frac{\omega t}{2}\right) .
\end{aligned}
$$

We focus on the evaluation of the photon number distribution $P(n, t)$, characterizing the dynamics of the state. A straightforward derivation shows that

$$
\begin{aligned}
P(n, t) & =|\langle g, n \mid \psi(t)\rangle|^{2}+|\langle e, n \mid \psi(t)\rangle|^{2} \\
& =\frac{1}{R}\left[\left|\vartheta_{t}\right|^{2} \cos ^{2}(\gamma t)+\left|\vartheta_{t+T_{B} / 2}\right|^{2} \sin ^{2}(\gamma t)\right]
\end{aligned}
$$

where $\left|\vartheta_{t}\right|^{2}$ has the explicit form

$$
\left|\vartheta_{t}\right|^{2}=\exp \left[-\alpha^{2}\left(n-n_{t}\right)^{2}\right],
$$

which represents a Gaussian distribution with time-dependent center $n_{t}$. It is shown that the photon number distribution $P(n, t)$ exhibits amplitude modulated BOs.

We note that $P(n, t)$ is directly determined by the factor $\gamma$ in Eq. (18), which depends on the ratio $g \sqrt{n} / \omega$. The numerical method is employed to simulate the time evolution process. It is performed by exact diagonalization of the Hamiltonian with truncated approximation. We are interested in the suppression and process of the probability transition. In the following, we will investigate the cases with two types of parameters: i) adjusting the ratio $g \sqrt{\bar{n}} / \omega$ for the zero-transition point, and ii) in-phase resonant oscillating $\Omega(t)$.

\subsection{Constant $\Omega$}

According to the approximate analysis, when we take $g \sqrt{\bar{n}} / \omega$ satisfying $\mathrm{J}_{0}(L)=0$, the transition between two BOs can be frozen. However, in practice a deviation may occur with respect to the approximate solution. We compute the time evolution and search the zero-transition point for the initial state in the form of Eq. (34). We consider four typical cases with $L=24.31,25.73,27.50$, and 28.89. From the plot of the function $\mathrm{J}_{0}(x)$ in Fig. (3), we can see that two of the four points locate at the vicinity of the zeros of the Bessel function, while the others locate at the stationary points. In Fig. (4), the photon number distributions $P(n, t)$ for these parameters are plotted. The corresponding analytical results $n_{t}$ (and $n_{t+T_{B} / 2}$ ) in Eq. (39) are also added on the plots of $P(n, t)$ as a comparison and guide. 
Amplitude modulated Bloch oscillations of photon probability distribution in cavity-atom system 11

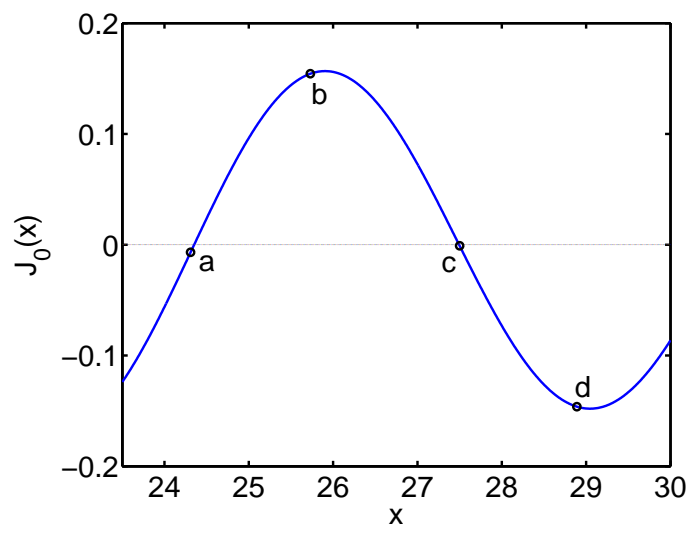

Figure 3. (Color online) Bessel function $\mathrm{J}_{0}(x)$. Two points a and c with $x=24.31$ and 27.50, are in the vicinity of the zeros. Points b and d with $x=25.73$ and 28.89, are the stationary points.

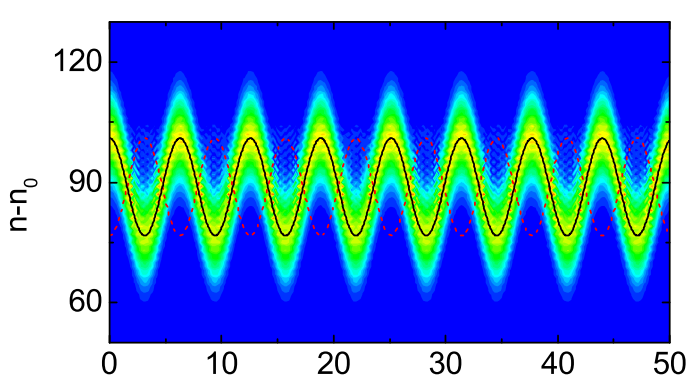

(a)

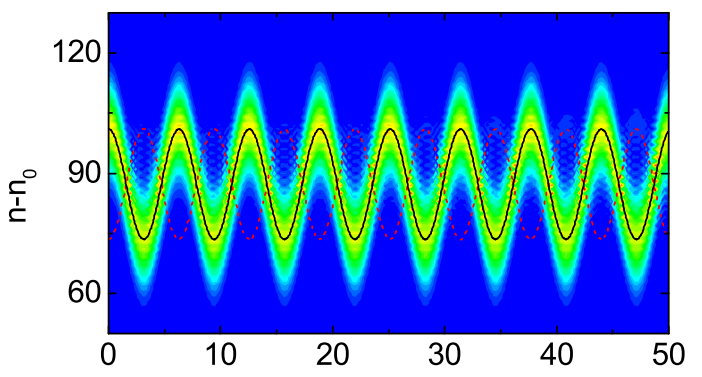

(c)

$\omega t$

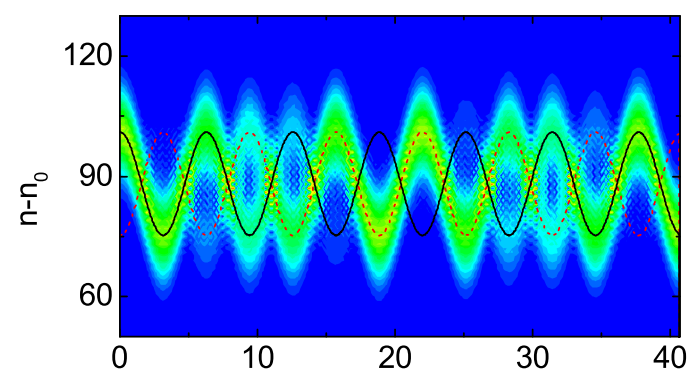

(b)

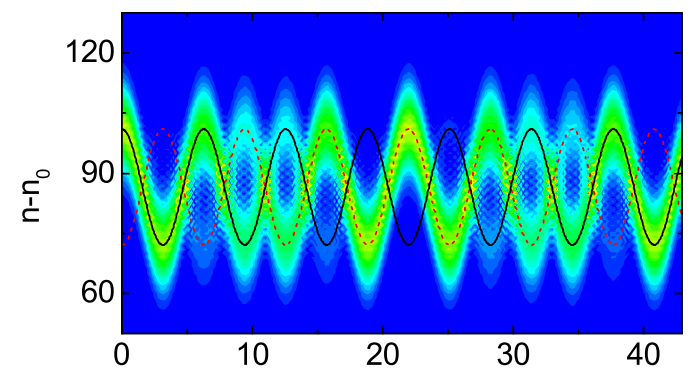

(d)

Figure 4. (Color online) Time evolution of the initial state in Eq. (34) with the same $\bar{n}$ and $\alpha$ as that in Fig. (2). It is driven by the original Rabi Hamiltonians with $\Omega=\omega$ and parameters (see Fig. (2)) (a) $L=24.31$, (b) 25.73, (c) 27.50, and (d) 28.89. The photon number distribution $P(n, t)$ is obtained by exact diagonalization. The solid and dashed lines are the center positions of two wavepackets from Eq. (39). It shows that the probability transition is frozen in the cases of (a) and (c). 


\subsection{In-phase resonant oscillating $\Omega(t)$}

We consider the resonant $\Omega(t)$, which have the half period as the Bloch oscillations. To clarify the action of $\Omega(t)$, we take a rectangular wave as

$$
\Omega(t)=\left\{\begin{array}{cc}
\omega, & \frac{1}{2}\left(n T-T_{1}\right)<\left|t-\varphi_{0}\right| \leq \frac{1}{2}\left(n T+T_{1}\right), \\
0 & (n=1,2,3 \ldots,) \\
& \text { otherwise }
\end{array}\right.
$$

with $T=T_{B}, T_{1}=0.1 T$. Here $\varphi_{0}$ control the phase between $\Omega(t)$ and the Bloch oscillations. We consider two typical cases with $\varphi_{0}=0$ and $T / 4$, which are in- phase and out-of-phase with the Bloch oscillations respectively.

Similarly, we can also take a sinusoidal wave as

$$
\Omega(t)=0.5\left\{1+\cos \left[2 \omega\left(t+\varphi_{0}\right)\right]\right\} \omega,
$$

with $\varphi_{0}=0$ and $T / 4$, respectively. Here we use a uniform mesh in the time discretization for a time-dependent Hamiltonian, i.e.,

$$
|\psi(t)\rangle=\exp \left[-i \int_{0}^{t} H\left(t^{\prime}\right) d t^{\prime}\right]|\psi(0)\rangle .
$$

The profiles of the evolutions with different types of $\Omega(t)$ are plotted in Fig. 5, The numerical result accords with our prediction: in the case of in-phase $\Omega(t)$, the wave dynamics is similar to the case with constant $\Omega$, while in the case of out-of-phase $\Omega(t)$, is similar to the case with zero $\Omega$.

\section{Summary}

In summary, we have studied the dynamics of the Rabi Hamiltonian, identifying a parameter regime corresponding to amplitude modulated BOs, another type of BlochZener oscillations. It is the first time to present a periodic phenomenon for this wellknown model when the coupling constant goes beyond the RWA. It also reveals a fact that a significant effect on the field, Bloch-Zener oscillation of the photon probability distribution with a distinct amplitude. It is remarkable that such a macroscopic phenomenon is induced by a single atom. Moreover, the probability transition between the two BOs can be controlled and suppressed by the ratio $g \sqrt{n} / \omega$, as well as the in-phase resonant oscillating atomic frequency $\Omega(t)$, leading to multiple zero-transition points. The numerical simulation of dynamics in the Rabi model confirms this prediction. However, the experimental observation of this prediction requires a coherent time scale of $g \sqrt{n}$, which is still a challenge so far. At the same time, with such a high number of photons in a quantum resonator, dissipation may have a role, too.

\section{Acknowledgments}

We acknowledge the support of the National Basic Research Program (973 Program) of China under Grant No. 2012CB921900 and CNSF (Grant No. 11374163). 

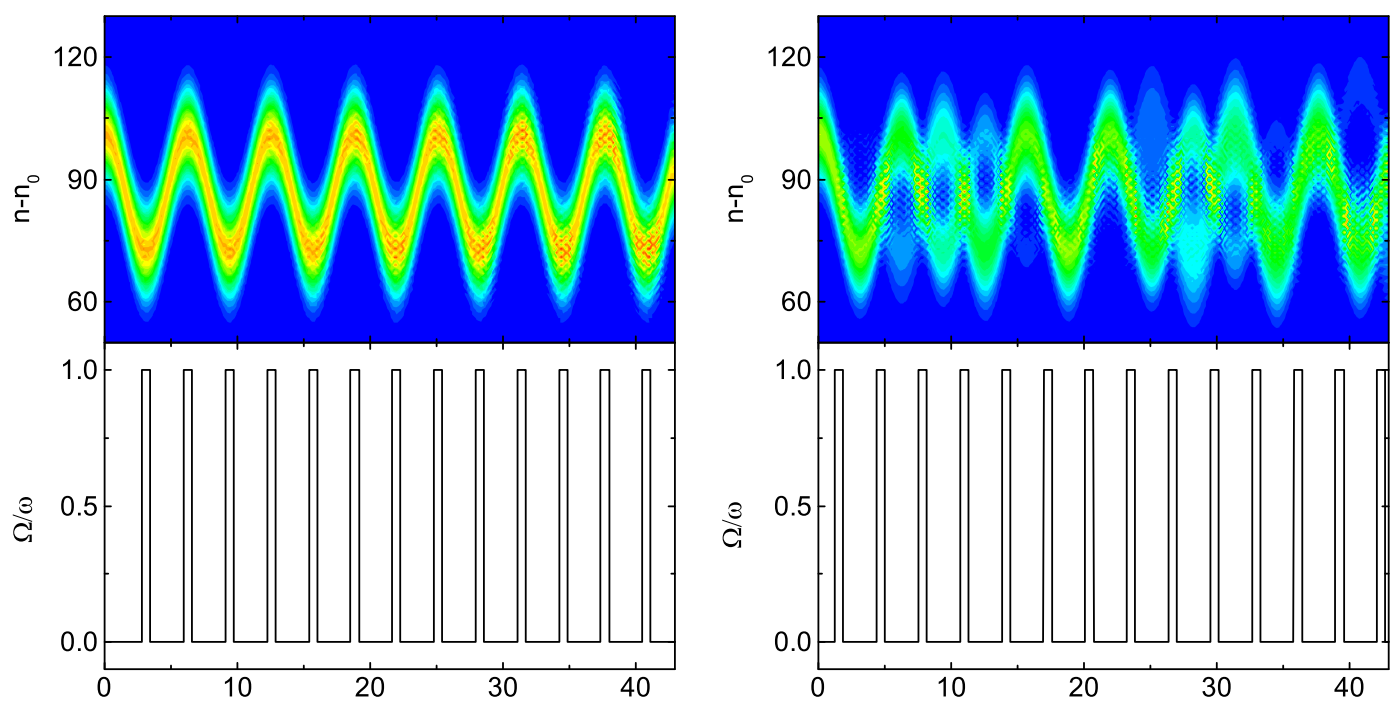

(a)

$\omega t$

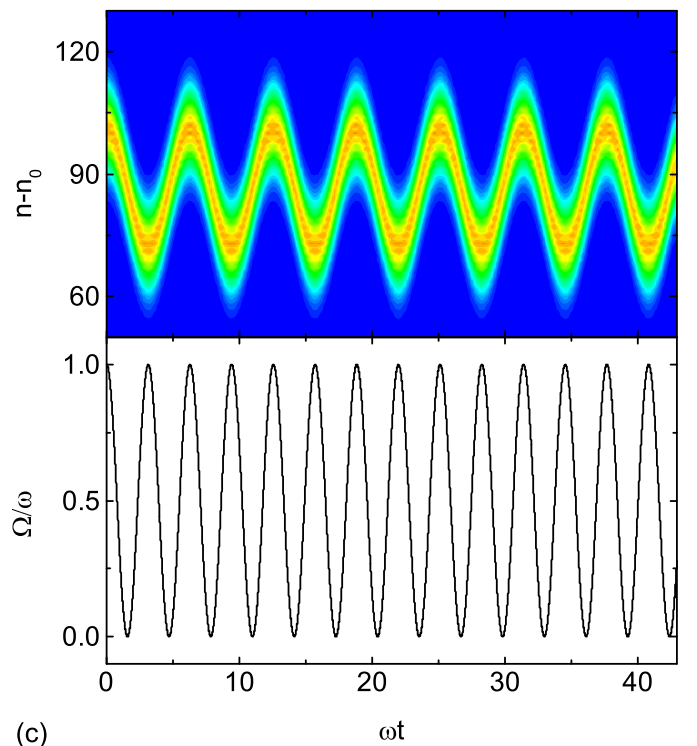

(b)

$\omega t$

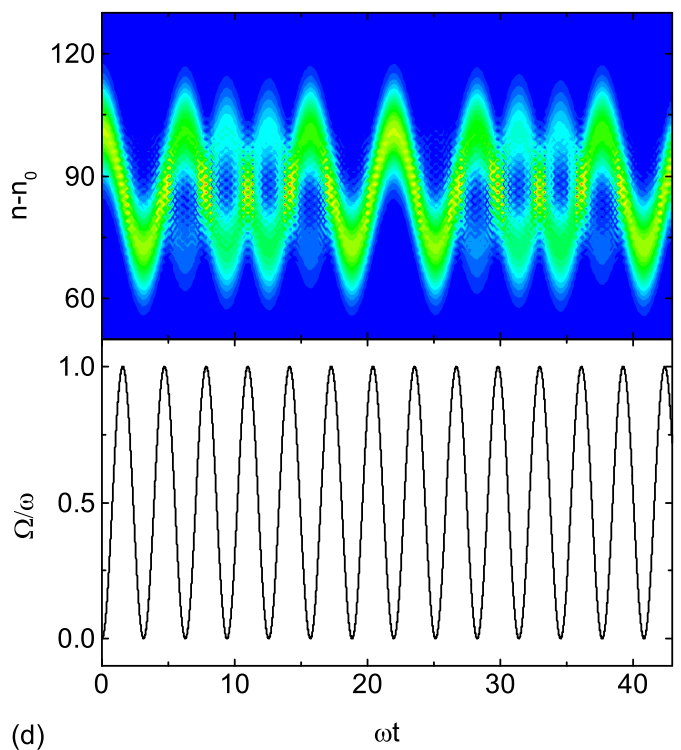

(d)

Figure 5. (Color online) Time evolution of the initial state in Eq. (34) with the same $\bar{n}$ and $\alpha$ as that in Fig. (2) $4 g \sqrt{n} / \omega=28.89$. The $\Omega(t)$ is taken in the form of Eq. (42) with (a) $\varphi_{0}=0$ and (b) $T / 4$, and in the form of Eq. (43) with (c) $\varphi_{0}=0$ and (d) $T / 4$. We can see that for both square and sinusoidal waves of $\Omega(t)$, the profile of the evolution in the case of in-phase (out-of-phase) is similar to the case with constant $\Omega$ (zero $\Omega$ ). This accords with our prediction. 
Amplitude modulated Bloch oscillations of photon probability distribution in cavity-atom system 14

\section{References}

[1] Rabi I I 1936 Phys. Rev. 49, 324

[2] Rabi I I 1937 Phys. Rev. 51, 652

[3] Nielsen M A and Chuang I L 2004 Quantum Computation and Quantum Information (Cambridge: Cambridge University Press)

[4] Braak D 2011 Phys. Rev. Lett. 107100401

[5] Solano E 2011 Physics 468

[6] Casanova J, Romero G, Lizuain I, García-Ripoll J J and Solano E 2010 Phys. Rev. Lett. 105 263603

[7] Jaynes E T and Cummings F W 1963 Proc. IEEE 5189

[8] Meystre P, Geneux E, Quattropani A and Faist A 1975 Nuovo Cimento B 25521

[9] Eberly J H, Narozhny N B and Sanchez-Mondragon J J 1980 Phys. Rev. Lett. 441323

[10] Narozhny N B, Sanchez-Mondragon J J and Eberly J H 1981 Phys. Rev. A 23236

[11] Englund D, Faraon A, Fushman I, Stoltz N, Petroff P and Vučković J 2007 Nature 450857 - 861

[12] Devoret M, Girvin S and Schoelkopf R 2007 Ann. Phys. (Leipzig) $16767-779$

[13] Ciuti C, Bastard G and Carusotto I 2005 Phys. Rev. B 72115303

[14] Pan F, Guan X, Wang Y and Draayerb J P 2010 J. Phys. B: At. Mol. Opt. Phys. 43175501

[15] Chen Q H, Wang C, He S, Liu T and Wang K L 2012 Phys. Rev. A 86023822

[16] Chilingaryan S A and Rodríguez-Lara B M 2013 J. Phys. A: Math. Theor. 46335301

[17] Ridolfo A, Leib M, Savasta S and Hartmann M J 2012 Phys. Rev. Lett. 109193602

[18] Felicetti S, Romero G, Rossini D, Fazio R and Solano E 2014 Phys. Rev. A 89013853

[19] Garcí-Ripoll J J, Peropadre B and De Liberato S 2014 arXiv:1410.7785

[20] Breid B M, Witthaut D and Korsch H J 2006 New J. Phys. 8110

[21] Breid B M, Witthaut D and Korsch H J 2007 New J. Phys. 962

[22] Witthaut D, Trimborn F, Kegel V and Korsch H J 2011 Phys. Rev. A 83013609

[23] Longhi S 2006 Europhys. Lett. 76416

[24] Longhi S 2008 Phys. Rev. Lett. 101193902

[25] Krueckl V and Richter K 2012 Phys. Rev. B 85115433

[26] Jaynes E T and Cummings F W 1963 Proc. IEEE 5189

[27] Shore B W and Knight P L 1993 J. Mod. Opt. 401195

[28] Niemczyk T et al 2010 Nature 6772

[29] Forn-Díaz P, Lisenfeld J, Marcos D, García-Ripoll J J, Solano E, Harmans C J P M and Mooij J E 2010 Phys. Rev. Lett. 105237001

[30] Higgins K D B, Lovett B W and Gauger E M 2013 Phys. Rev. B 88155409

[31] Fukuyama H, Bari R A and Fogedb y H C 1973 Phys. Rev. B 85579

[32] Hartmann T, Keck F, Korsch H J and Mossmann S 2004 New J. Phys. 62

[33] Glück M, Kolovsky A R and Korsch H J 2002 Phys. Rep. 366103

[34] Bloch F 1928 Z. Phys. 52555

[35] Zener C 1934 Proc. R. Soc. A 145523

[36] Lyssenko V G, Valusis G, Löser F, Hasche T and Leo K 1997 Phys. Rev. Lett. 79301

[37] Cristiani M, Morsch O, Müller J H, Ciampini D and Arimondo E 2002 Phys. Rev. A 65063612 